\title{
Terlipressina: profilo farmacologico clinico, terapeutico e farmacoeconomico nel trattamento delle varici esofagee
}

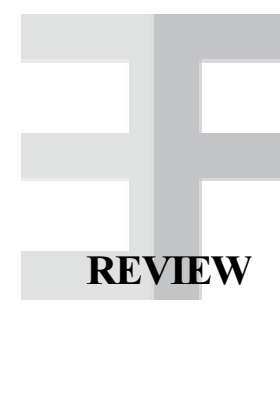

Lorenzo Pradelli*

\begin{abstract}
The formation and rupture of esophageal and gastric varices are severe but common complications of hepatic cirrhosis. They represent the cause underlying more than one forth of all deaths in cirrhotic patients and exact an impressive burden in terms of survival, quality of life and health care resource consumption.

Therapeutic options for the control of active variceal hemorrhage comprise pharmacological agents, endoscopic techniques and surgery. Of these, the only options immediately available in the absence of highly specialized medical personnel are vasoactive drugs, namely vasopressin and derivatives and somatostatin and derivatives. Despite this clear advantage, the role of pharmacological treatment is still debated; furthermore, there's no consensus on the drug of choice for variceal bleeding control.

In this paper the main pharmacological and clinical features of terlipressin, a synthetic vasopressin analog, are outlined, as well as a frame for its pharmacoeconomical evaluation. Terlipressin is the only vasoactive drug that demonstrated survival benefits in cirrhotic patients with active variceal hemorrhage, even in those treated with endoscopic sclerotherapy, and is characterized by a favorable risk/benefit ratio.

The relative cost-effectiveness of the different drugs, nevertheless, is yet to be determined by methodologically rigorous studies, partly because at the present state of the research there still are clinical and economical uncertainties to be cleared.
\end{abstract}

Keywords: terlipressin, variceal hemorrhage, economical impact

Farmeconomia e percorsi terapeutici 2005; 6 (1): 57-68

\section{INTRODUZIONE}

La formazione di varici esofagee e gastriche è una grave ma comune conseguenza dell'ipertensione portale, dovuta nella maggioranza dei casi a cirrosi epatica. Esse sono riscontrabili in circa il $30 \%$ dei pazienti cirrotici ben compensati e nel $60 \%$ di quelli con cirrosi scompensata [1]. Il rischio di rottura è circa del $10-20 \%[1,2]$ per anno se vengono considerati tutti i pazienti con varici, superiore al $20-30 \%$ nei soggetti con varici di grandi dimensioni [3]. La rottura di varici esofagee è responsabile di oltre un quarto dei decessi nei pazienti cirrotici [4]: nel 30-50\% dei casi il primo episodio di emorragia da varice è letale [1], circa un terzo dei sopravvissuti presenta una recidiva entro i 6 mesi dal primo episodio e due terzi entro i 2 anni, con tassi di mortalità del $30-50 \%$ per ogni episodio $[5,6,7]$. Oltre al costo in termini di sopravvivenza, gli episodi emorragici a carico delle varici esofagee $o$ gastriche comportano un elevatissimo consumo di risorse sanitarie: il paziente con sanguinamento atti- vo deve essere ricoverato, normalmente in reparti di terapia intensiva, richiede spesso la trasfusione di prodotti ematici, necessita di terapia farmacologica e/o endoscopica e di successive endoscopie di controllo.

Coerentemente, le stime sui costi diretti e indiretti delle varici esofagee sono eclatanti: nel 1985 i costi sanitari diretti sostenuti per la gestione di questi pazienti negli USA sono stati calcolati in 78,5 milioni di dollari, cui andavano sommate le spese per 62.000 giornate di degenza ospedaliera e 47,5 milioni di dollari di costi indiretti [8]. Sempre in quell'anno, la rottura di varici esofagee è stata la più onerosa delle patologie gastrointestinali in termini di costo medio per giornata di ospedalizzazione, pari a oltre 1.000 US\$ del 1985.

Le opzioni terapeutiche in grado di controllare l'emorragia acuta comprendono trattamenti farmacologici, meccanici, endoscopici e chirurgici (tabella I). Di queste, le uniche strategie attuabili rapidamente da personale non altamente specializzato sono quelle farmacoterapiche. Nonostante questo eviden- 
te vantaggio, vi è ancora notevole disaccordo tra i vari specialisti sia sul ruolo della farmacoterapia, sia sulla scelta del farmaco da utilizzare, sia sulla complementarità con le altre tecniche disponibili. Le classi di farmaci che si sono rivelate utili nella gestione dei pazienti con varici esofagee sono in grado di ridurre il flusso afferente alla porta, mediante costrizione del letto vascolare splancnico, o di ridurre la resistenza intraepatica: i beta-bloccanti, che però hanno un ruolo esclusivamente profilattico (sia come prevenzione primaria sia come secondaria, per ridurre il rischio di recidiva nei sopravvissuti), somatostatina e analoghi, vasopressina e analoghi, e nitrati organici, i soli ad agire sulla resistenza vascolare intraepatica. L'unico intervento farmacologico che si è dimostrato in grado di ridurre significativamente la mortalità associata al sanguinamento da rottura di varici esofagee è la somministrazione precoce di terlipressina, un analogo sintetico della vasopressina, anche in associazione alla scleroterapia, se quest'ultima è disponibile e praticabile d'urgenza.

In questo lavoro presentiamo una rassegna di studi clinici ed economici che hanno valutato l'utilizzo della terlipressina nel controllo delle emorragie da varici esofagee e di altre complicazioni della cirrosi epatica, per contribuire a definirne il corretto ambito di utilizzo razionale e costo-efficace nei pazienti cirrotici.

\section{PROFILO FARMACOLOGICO TERAPEUTICO}

\section{Meccanismo d'azione e farmacodinamica}

Lo sviluppo di varici gastriche ed esofagee consegue all'ipertensione portale, quando questa raggiunge valori elevati: si ritiene che lo sviluppo di varici sia probabile a livelli di gradiente pressorio porto-sistemico (la differenza tra la pressione vigente nella vena porta e quella mi- surata nella cava inferiore) superiori a $10 \mathrm{mmHg}$. Quando il gradiente porto-sistemico supera i 12 $\mathrm{mmHg}$, il paziente è esposto ad un alto rischio di emorragia da rottura di varici $[9,10]$.

In presenza di cirrosi epatica, l'ipertensione portale è conseguenza di due ordini principali di fattori, che si verificano contemporaneamente: aumento del flusso splancnico e portale, dovuto alla liberazione di vasodilatatori (sono stati chiamati in causa glucagone, VIP, sostanza P, prostaciclina, acidi biliari, TNF-alfa e NO) [11] e aumentata resistenza al flusso venoso sinusoidale intraepatico, causato dall'alterazione dell'architettura parenchimale, con compressione delle venule da parte di noduli in fase di proliferazione, da aumentata deposizione di collageno negli spazi di Disse e da livelli intraepatici elevati di vasocostrittori ad azione locale. Il miglioramento di questi fattori è in grado di ridurre la pressione portale e di arrestare 0 prevenire l'emorragia da rottura di varice [10].

Terlipressina è un derivato sintetico della vasopressina, caratterizzato da una catena laterale contenente tre molecole di glicil-lisina. Sebbene la molecola immodificata possieda una debole attività intrinseca, terlipressina si comporta essenzialmente da pro-farmaco, liberando lipressina in seguito a trasformazione enzimatica, un processo che perdura mediamente 4-6 ore dopo la somministrazione [12]. L'azione farmacologica principale consiste nell'aumento di tono della muscolatura liscia, vascolare e non, particolarmente a livello gastrointestinale, con conseguente vasocostrizione, riduzione del flusso splancnico e della pressione portale. A queste modificazioni nella distribuzione del flusso corrispondono, a livello emodinamico, un lieve aumento delle pressioni arteriose sistolica e diastolica e una riduzione della frequenza cardiaca. Queste azioni sono qualitativamente paragonabili a quelle della vasopressina, ma se ne differenziano per la minore intensità e la maggiore durata, probabilmente dovute al rila-
Tabella I

Opzioni terapeutiche per la gestione delle emorragie da rottura di varici esofagee

\begin{tabular}{llcl}
\hline \multicolumn{1}{c}{ Farmaci } & Mezzi meccanici & Endoscopia & Chirurgia \\
\hline Terlipressina & $\begin{array}{l}\text { Tamponamento con } \\
\text { sonda a palloncino }\end{array}$ & Scleroterapia & $\begin{array}{c}\text { Shunt porto-sistemico } \\
\text { intraepatico per via } \\
\text { transgiugulare (TIPS) }\end{array}$ \\
Vasopressina & $\begin{array}{l}\text { Tamponamento con } \\
\text { sonda a palloncino }\end{array}$ & Shunt porto-sistemico \\
Somatostatina & $\begin{array}{l}\text { Tamponamento con } \\
\text { sonda a palloncino }\end{array}$ & & $\begin{array}{c}\text { Devascolarizzazione } \\
\text { esofagea }\end{array}$ \\
Octreotide & $\begin{array}{l}\text { Tamponamento con } \\
\text { sonda a palloncino }\end{array}$ & Legatura delle varici & Trapianto di fegato \\
Beta-bloccanti & $\begin{array}{l}\text { Tamponamento con } \\
\text { sonda a palloncino }\end{array}$ & Legatura delle varici & \\
\hline
\end{tabular}


scio protratto di lisin-vasopressina ad opera delle eso- ed endopeptidasi localizzate a livello epatico e renale $[13,14]$. Tutti gli effetti emodinamici sistemici sono reversibili con la somministrazione di nitrati organici [15].

Gli effetti emodinamici di terlipressina sono stati valutati specificatamente nei pazienti cirrotici $[15,16,17]$, alla luce dell'utilizzo pratico della molecola, destinata eminentemente a questa tipologia di pazienti. A parte le risposte pressoria e bradicardica su accennate, è stata osservata una riduzione dei flussi epatico e splenico, con riduzione della pressione intravariceale valutata intorno al $25 \%$ dei valori pre-trattamento. In uno studio randomizzato e controllato di Nevens e coll. [18], gli effetti sulla pressione intravariceale di terlipressina ( $2 \mathrm{mg}$ ) sono stati confrontati in 30 pazienti con quelli di octreotide $(50 \mathrm{mg})$ e di un placebo. Terlipressina ha prodotto diminuzioni significative della pressione sanguigna all'interno delle varici esofagee a partire da 2 minuti dalla somministrazione endovenosa, mentre placebo e octreotide non hanno mostrato tali effetti in misura coerente e significativa.

\section{Farmacocinetica, vie di somministrazione e posologie}

Terlipressina viene normalmente somministrata per bolo lento endovenoso, ma viene assorbita anche per le vie intranasale e endobronchiale $[13,19]$, che tuttavia rivestono scarso interesse pratico. In seguito all'iniezione, terlipressina si distribuisce rapidamente nel plasma, seguendo una cinetica di secondo ordine con un tempo medio di distribuzione di 8-9 minuti e un volume apparente di 0,6-0,9 L/kg di peso corporeo [14]. L'attivazione metabolica, come già accennato, avviene ad opera di peptidasi tissutali localizzate prevalentemente a livello epatico e renale. Il processo enzimatico responsabile è la scissione di molecole di lisin-vasopressina (lipressina) dalla terlipressina ed è mantenuto per circa 4-6 ore dalla somministrazione. All'equilibrio, raggiunto dopo circa un'emivita di terlipressina (50-60 minuti), le concentrazioni di lipressina sono approssimativamente un ventesimo di quelle di terlipressina. L'eliminazione di terlipressina e lipressina è prevalentemente metabolica, ad opera di peptidasi tissutali, mentre la quota eliminata con le urine in forma non modificata è minore, stimata intorno all' $1-2 \%$ della dose somministrata.

La posologia raccomandata per la gestione delle emorragie da rottura di varici esofagee è di $2 \mathrm{mg}$, da somministrarsi come bolo endovenoso lento ogni 4-6 ore, fino all'arresto dell'emorragia. Una volta ottenuto il controllo del sanguinamento, il trattamento viene in genere continuato per un massimo di ulteriori 24 ore, a dosaggi ridotti.

\section{Efficacia clinica}

\section{A) Emorragia da rottura di varici esofagee}

L'efficacia di terlipressina nel trattamento delle varici esofagee sanguinanti è stata oggetto di numerosi studi di valutazione, condotti sia contro placebo, sia contro le altre alternative disponibili. Questi lavori sono stati approfonditamente e rigorosamente analizzati da Ioannou et al. [20] in una recente review sistematica, in cui sono state condotte metaanalisi su tutti i dati omogenei provenienti da studi randomizzati e controllati. Vista l'elevata qualità e attualità della review, che ha incluso anche una valutazione sull'appropriatezza del disegno e dei metodi impiegati dai vari ricercatori, nell'esposizione dell'efficacia clinica di terlipressina faremo spesso riferimento ai dati ottenuti da questo gruppo di lavoro.

\section{Studi controllati contro placebo}

La meta-analisi di Ioannou e coll. ha identificato sette studi randomizzati e controllati contro placebo, condotti su un totale di 443 pazienti con emorragia da rottura di varici esofagee. L'analisi dei dati aggregati ha messo in evidenza che la somministrazione di terlipressina riduce in maniera statisticamente significativa il rischio di fallimento iniziale dell'emostasi (RR: 0,66, IC 95\% 0,53 - 0,82), di dover ricorrere a procedure strumentali per arrestare l'emorragia iniziale o il risanguinamento (RR: 0,72, IC 95\% 0,55-0,93) e, soprattutto, di morte (RR: 0,66, IC 95\% 0,49-0,88). Quest'ultimo dato acquisisce ancora maggiore importanza alla luce dell'osservazione che terlipressina è l'unico trattamento farmacologico che si è dimostrato in grado di migliorare la sopravvivenza dopo emorragia da varici. L'outcome di mortalità impiegato nella review è inoltre particolarmente robusto, in quanto è stato deciso di considerare l'emorragia come causa diretta di ogni decesso registrato nei 42 giorni successivi all'evento, in accordo con le raccomandazioni del III Baveno consensus workshop, tenutosi nel 2000.

Abbiamo accennato alla valutazione della qualità degli studi compresa nella review: essa si è basata sulla scala di Jadad, che permette di distinguere gli studi ad alta qualità (pochi bias) da quelli a bassa qualità (alti bias) sulla base del disegno, dei metodi e della completezza dell'informazione contenuta nei report. La metaanalisi condotta su tutti gli studi di confronto con placebo è stata ripetuta includendo unicamente i risultati dei lavori di elevata qualità (3 su 7), per escludere che gli studi a bassa qualità fossero i principali driver delle differenze osservate. Questa analisi ha confermato i risultati della valutazione principale: l'utilizzo di terlipressina è associato a riduzioni statistica- 
mente significative della mortalità (RR 0,61, IC $95 \% 0,45-0,84)$, del rischio di fallimento dell'emostasi (RR 0,66, IC 95\% 0,52-0,83) e della necessità di ricorrere a procedure strumentali per arrestare emorragie incontrollate 0 recidivanti (RR 0,68, IC 95\% 0,52-0,90). Poiché il protocollo di tre dei sette studi presi in considerazione per la meta-analisi prevedeva l'esecuzione di una scleroterapia endoscopica al momento dell'inclusione, gli autori della review hanno condotto un'ulteriore meta-analisi su questi dati, al fine di verificare se la somministrazione di terlipressina potesse migliorare l'efficacia della scleroterapia.

L'effetto favorevole del farmaco è risultato relativamente inferiore negli studi su pazienti trattati endoscopicamente con scleroterapia, ma anche in questa popolazione di pazienti l'aggiunta di terlipressina è risultata associata a benefici statisticamente significativi (mancato controllo iniziale dell'emorragia) o prossimi alla significatività.

Tutti i sette studi randomizzati e controllati contro placebo hanno dimostrato che l'utilizzo di terlipressina è associato a un minor fabbisogno di trasfusioni di sangue, mentre nessun autore ha riportato i dati sulla durata del ricovero ospedaliero [20].

\section{Studi di confronto con vasopressina}

La vasopressina, o ormone antidiuretico $(\mathrm{ADH})$, è un ormone naturale secreto dal lobo posteriore della ghiandola pituitaria, la cui funzione principale consiste nel contributo al mantenimento dell'omeostasi idro-salina. Il suo impiego nella cirrosi sfrutta però un'altra azione, quella vasocostrittrice a livello splancnico, evocata dal legame dell'ormone con il recettore V1 sulla muscolatura liscia vascolare, in particolare nel distretto arterioso mesenterico.

La vasopressina, sotto forma di "estratto di ipofisi posteriore", viene somministrata ai pazienti con emorragia da rottura di varice esofagea fin dagli anni 50-60 [10]. Svariati studi indicano un tasso di controllo dell'emorragia acuta intorno al $29-71 \%$ quando impiegata in monoterapia e del $45-73 \%$ in associazione con nitrati organici: la maggior parte degli studi controllati dimostra un'efficacia superiore al placebo e un ulteriore incremento del tasso di successo con l'aggiunta dei nitroderivati [1].

In uno studio di confronto terlipressina vs. vasopressina $(0,4$ unità/minuto per infusione ev continua) controllato contro placebo e condotto nel 1982 da Freeman su 19 pazienti con emorragia da varici esofagee, terlipressina ( $2 \mathrm{mg}$ ev ogni 6 ore) è stata in grado di controllare il sanguinamento nel $70 \%$ dei pazienti, contro il $9 \%$ dei pazienti che hanno ricevuto vasopressina, i quali, inoltre, hanno avuto bisogno di più trasfusioni di sangue [21]. L'efficacia di vasopressina in questo studio, tutta- via, è insolitamente bassa; la maggior parte degli studi pubblicati, come accennato più sopra, ne attesta un valore vicino al $50 \%$.

Dalla meta-analisi di Ioannou e coll., che ha incluso i dati di 301 pazienti arruolati in 5 studi, non emergono differenze significative tra vasopressina e terlipressina per nessuno dei parametri di efficacia valutati [20].

\section{Studi di confronto con somatostatina}

La somatostatina è un ormone peptidico prodotto a livello ipotalamico e gastrointestinale, con funzione prevalente di modulazione del rilascio di altri ormoni, tra cui $\mathrm{GH}$, insulina, glucagone, colecistochinina, VIP e altri. L'utilità nel controllo delle emorragie da varici esofagee è probabilmente dovuta proprio all'azione inibitoria su alcuni di questi ormoni che inducono vasodilatazione splancnica, in quanto la somatostatina non possiede azioni vasoattive intrinseche [22]. La somatostatina, così come la vasopressina, deve essere somministrata in infusione lenta, in virtù della brevissima emivita plasmatica, valutata intorno al minuto.

Sono stati condotti tre studi randomizzati per il confronto di terlipressina e somatostatina nella gestione delle emorragie da rottura di varici. Né i risultati dei singoli studi, né la metaanalisi che ha aggregato i dati dei 302 pazienti arruolati sono stati in grado di dimostrare alcuna differenza di efficacia statisticamente significativa tra le due opzioni terapeutiche [20].

\section{Studi di confronto con octreotide}

Octreotide è un analogo sintetico della somatostatina, caratterizzato da un'emivita plasmatica allungata a 10-22 minuti. Il tempo di permanenza in circolo rimane tuttavia piuttosto breve, per cui nella maggioranza dei casi viene anch'esso somministrato per infusione continua, per quanto possa anche essere utilizzata la via sottocutanea.

Il meccanismo d'azione ipotizzato è il medesimo di quello della somatostatina, così come l'efficacia, che si assesta su valori del 40-80\% [23-26]. La somministrazione in bolo di octreotide è risultata associata a riduzione della gittata cardiaca e aumento della pressione di incuneamento polmonare, per quanto la rilevanza clinica di tali modificazioni emodinamiche non sia chiara [27]. Altri effetti collaterali sono stati descritti raramente con l'uso di octreotide e somatostatina.

La review di Ioannou ha identificato tre studi di bassa qualità, condotti nel complesso su 203 pazienti, che hanno confrontato octreotide e terlipressina nell'emorragia da varice esofagea. La meta-analisi non ha dimostrato differenze significative in termini di mortalità, recidive e ricorso a procedure strumen- 
tali per il controllo dell'emorragia, mentre ha messo in luce una frequenza di fallimento dell'emostasi di prima battuta significativamente maggiore nel gruppo dei pazienti che hanno ricevuto terlipressina. Anche in questo caso, non sono state registrate o riportate le durate dei ricoveri ospedalieri [20].

\section{Studi di confronto con procedure endoscopiche}

Le principali misure terapeutiche attuabili per via endoscopica in presenza di varici esofagee sono la legatura con elastico, la sclerotizzazione con sostanze iniettate durante l'esame endoscopico e il tamponamento con catetere a palloncino. Di queste, solo le ultime due sono opzioni valide in casi di emorragia acuta e attiva della varice.

Ioannou e coll. hanno incluso nella loro review tre studi randomizzati che hanno confrontato il tamponamento della varice sanguinante con catetere a palloncino e il trattamento vasoattivo a base di terlipressina, senza evidenziare differenze statisticamente significative negli outcome di efficacia adottati [20]

Nemmeno il confronto tra terlipressina e scleroterapia d'urgenza effettuata per via endoscopica, svolto in uno studio clinico randomizzato condotto su 219 pazienti, ha messo in evidenza differenze significative negli esiti a breve o lungo termine [28].

\section{B) Sindrome epato-renale}

La sindrome epato-renale (SER) colpisce circa il $20 \%$ dei pazienti con cirrosi epatica e ascite, ed è caratterizzata da intensa vasocostrizione renale, basso tasso di filtrazione glomerulare, oliguria e ritenzione salina in presenza di funzione tubulare integra e istologia normale. In base alla presentazione clinica vengono distinte due forme: nella SER di tipo I il deterioramento della funzione renale è rapido e la mortalità prossima al $100 \%$, mentre la SER di tipo II insorge più lentamente $\mathrm{e}$ può essere reversibile con il trapianto di fegato o con un intervento di shunt porto-sistemico transgiugulare intraepatico (TIPSS).

La somministrazione di terlipressina, particolarmente se associata ad albumina, appare in grado di correggere o rallentare lo sviluppo della SER di tipo II, anche se la casistica pubblicata è ancora limitata. Le prime segnalazioni in merito riscontrabili in letteratura sono due case-report del 1996 e 1997, riguardanti la gestione di due donne affette da epatopatia alcolica e funzione renale in declino [29,30]. La somministrazione di terlipressina ha migliorato la funzionalità renale in maniera rapida e stabile; in una delle due pazienti la terapia è stata mantenuta fino al momento del trapianto di fegato, nell'altro caso la terapia è stata sospesa a causa di segni di ischemia epicardica, ma la funzionalità renale è rimasta corretta ad un controllo effettuato dopo un mese dalla sospensione.

Nel 1998 Hadengue et al. hanno condotto uno studio in cross-over su 9 pazienti, trattati per 2 giorni con $2 \mathrm{mg}$ di terlipressina e, dopo 24 ore di wash-out, per altri due con placebo, in ordine casuale. In questo studio, la somministrazione di terlipressina è stata in grado di migliorare la filtrazione glomerulare: la clearance della creatinina è aumentata significativamente di più che con il placebo ( 27 vs 16 $\mathrm{ml} / \mathrm{min}, \mathrm{p}<0,05)$, così come la diuresi. Terlipressina, inoltre, ha ridotto le concentrazioni reniniche plasmatiche e ha aumentato l'escrezione di sodio in 6 dei 9 pazienti, mentre il placebo non ha avuto alcun effetto su questi parametri [31].

I benefici sull'insufficienza renale in corso di epatopatia appaiono ancora maggiori qualora venga associato un trattamento con albumina, come nello studio di Uriz e coll. dell'università di Barcellona. Terlipressina è stata somministrata alla dose iniziale di $0,5 \mathrm{mg}$ ogni 4 ore, poi incrementata in maniera graduale ogni 3 giorni, fino ad arrivare a 1 o $2 \mathrm{mg}$ ogni 4 ore in base alla risposta del paziente; i pazienti hanno inoltre ricevuto albumina $1 \mathrm{~g} / \mathrm{kg}$ il giorno 1 , poi da 20 a 40 grammi al dì nei giorni successivi, fino alla correzione della SER (creatinina sierica $<1,5 \mathrm{mg} / 100 \mathrm{ml}$ ) o per un massimo 15 giorni. Questo schema è stato in grado di correggere la SER in 7 pazienti su 9 e di consentire a 3 su 5 candidati di arrivare al trapianto di fegato: nel complesso il trattamento ha significativamente ridotto la creatinina sierica $(\mathrm{p}<0,001)$, triplicando il tasso di filtrazione glomerulare $(p<0,01)$ senza causare alcun effetto indesiderato grave [32].

Lo stesso gruppo di lavoro ha condotto un altro studio di valutazione della terlipressina nella terapia della SER, confrontando in $21 \mathrm{pa-}$ zienti l'approccio descritto sopra con la somministrazione di terlipressina senza aggiunta di albumina. Nel complesso, la terapia è stata in grado di indurre risposta completa in 12 pazienti (57\%), ma con notevoli differenze tra il gruppo ricevente anche albumina $(77 \%)$ e quello trattato in monoterapia $(25 \%, \mathrm{p}<0,03$ per il confronto). Nei pazienti che hanno ricevuto anche albumina, a differenza che in quelli trattati con la sola terlipressina, si sono osservati effetti favorevoli anche in termini aumento della pressione arteriosa e soppressione del sistema renina-angiotensina. Tra i soggetti che hanno mostrato una risposta completa al trattamento la recidiva è risultata rara $(17 \%)$ e la sopravvivenza migliore [33].

Un lavoro condotto presso l'Ospedale San Giovanni Battista di Torino suggerisce un'utilità anche diagnostica, oltre che terapeutica, 
della somministrazione di terlipressina ai soggetti cirrotici con ipertensione portale e insufficienza renale. In questo studio, infatti, un miglioramento degli indici di filtrazione glomerulare dopo somministrazione di terlipressina si è osservato solamente nei pazienti con disfunzione renale dovuta a sindrome epato-renale, e non in quelli con insufficienza renale da nefropatia organica, e tale risposta è risultata predittiva del successo associato all'intervento di TIPSS. Un tentativo terapeutico con terlipressina, oltre a essere di per sé risolutivo in alcuni casi, può dunque essere una valida strategia per la selezione di cirrotici nefropatici canditati alla TIPSS, poiché fornisce l'informazione clinica cruciale sulla reversibilità (o meno) della disfunzione renale [34] .

Un recente lavoro tedesco, infine, conferma l'efficacia di terlipressina nella terapia della SER anche in associazione a gelatinpolisuccinato, un sostituto del plasma dal costo notevolmente inferiore all'albumina. I sette pazienti arruolati in questo studio pilota sono stati trattati per 6 giorni, con notevoli miglioramenti dell'emodinamica (pressione arteriosa media $58+/-4.4 \mathrm{mmHg}$ vs. $75+/-4.5 \mathrm{mmHg}, \mathrm{p}<$ 0.001 ) in tutti e della funzionalità renale (creatinina sierica $3.85+/-0.44 \mathrm{mg} / \mathrm{dl} \mathrm{vs} .1 .9+$ / $-0.32 \mathrm{mg} / \mathrm{dl} ; \mathrm{p}<0.018$ ) in cinque [35].

\section{C) Cirrosi e gastropatia ipertensiva}

La riduzione della pressione portale è desiderabile anche nei pazienti che presentano cirrosi e/o iperemia gastrica secondaria all'ipertensione portale. Terlipressina è stata valutata anche in questi pazienti, con buoni risultati.

In uno studio in doppio cieco, 28 pazienti cirrotici con gastropatia da ipertensione portale hanno ricevuto $2 \mathrm{mg}$ di terlipressina $(\mathrm{n}=9)$, $0,4 \mathrm{U} / \mathrm{Min}$ di vasopressina $(\mathrm{n}=9)$ o placebo $(n=10)$. La risposta è stata valutata in termini di perfusione della mucosa gastrica, misurata con la spettrofotometria di riflettanza, un metodo per la stima del contenuto in emoglobina e ossigeno, e con la flussimetria laser-Doppler, che valuta la perfusione tissutale.

Vasopressina e terlipressina, a differenza del placebo, hanno causato un significativo aumento della pressione arteriosa e una diminuzione della frequenza cardiaca; questi effetti sono apparsi più pronunciati con vasopressina.

Vasopressina e terlipressina hanno determinato una riduzione duratura e simile della perfusione misurata con la flussimetria laserdoppler $(-36 \%+/-8 \%$ e $-34 \%+/-6 \%$, rispettivamente; $\mathrm{p}<0.05$ sia rispetto al basale sia rispetto al placebo), riducendo al contempo l'ossigenazione della mucosa gastrica; tale effetto, tuttavia, è risultato significativamente $(p<0,05)$ meno rilevante con terlipressina che con vasopressina. Con terlipressina, la caduta della perfusione si è verificata rapidamente, entro 2 minuti dalla somministrazione, mentre con vasopressina la riduzione ha avuto un andamento progressivo che non ha raggiunto il plateau nel corso degli 8 minuti di osservazione. Gli autori dello studio concludono che entrambi i farmaci sono in grado di ridurre efficacemente l'iperemia gastrica da ipertensione portale nei cirrotici e che la minor tendenza alla riduzione dell'ossigenazione gastrica osservata con terlipressina ha il potenziale di ridurre l'incidenza di eventi ischemici associata all'utilizzo di vasopressina [36].

\section{Tollerabilità nel paziente cirrotico}

Le principali reazioni avverse segnalate dopo somministrazione di terlipressina sono legate ai suoi meccanismi d'azione: contrazione della diuresi da una parte e manifestazioni da vasocostrizione, come dolore addominale, ipertensione arteriosa, dolori stenocardici, dall'altra.

Nella casistica presa in considerazione per la valutazione della sicurezza dalla meta-analisi di Ioannou, nessuna differenza nel numero di morti attribuibili a eventi avversi è stata rilevata tra i pazienti trattati con terlipressina e gli altri gruppi (placebo, catetere a palloncino, trattamento endoscopico e altri farmaci vasoattivi): 6 morti su 798 pazienti vs. 5 su 811 , rispettivamente.

Nessuna differenza è emersa nemmeno nel numero di eventi avversi che hanno provocato l'interruzione della terapia tra terlipressina e gli altri approcci, con l'eccezione di vasopressina. Nel gruppo terlipressina ci sono state 5 interruzioni del trattamento su 693 pazienti valutabili ( 3 casi attribuiti a bradicardia, uno a fibrillazione atriale e l'ultimo a insufficienza respiratoria), nei gruppi di confronto vi sono state 11 interruzioni del trattamento su 697 pazienti: 3 ulcere da compressione con il catetere a palloncino e 8 casi trattati con vasopressina, di cui un'ischemia miocardica, una cefalea grave, tre casi di dolore addominale, uno di bradicardia e due ipertensioni arteriose. L'analisi di confronto della tollerabilità di vasopressina vs. terlipressina ha evidenziato una frequenza significativamente superiore di eventi avversi di entità tale da indurre la sospensione del trattamento nei pazienti trattati con vasopressina $(p<0,05)[20]$.

Anche dal punto di vista della compatibilità con altri farmaci la terlipressina possiede un ottimo profilo di sicurezza: non sono stati segnalati casi di reazioni avverse attribuite a interazioni farmacocinetiche 0 farmacodinamiche. Inoltre, uno studio ha valutato la possibilità di associare terlipressina al trattamento profilattico con beta-bloccanti, per sondarne l'eventuale effetto sinergico sul 
tono portale [37]. In questi pazienti, l'aggiunta di terlipressina è stata in grado di diminuire la pressione portale senza indurre alterazioni emodinamiche sistemiche rilevanti, dimostrando che l'associazione beta-bloccante terlipressina è un'opzione sicura ed efficace per la correzione dell'ipertensione portale nei pazienti cirrotici.

\section{PROFILO FARMACOECONOMICO}

Il notevole impatto sociale ed economico delle varici esofagee ha indotto a svolgere una considerevole mole di ricerca anche in campo economico sanitario, benché l'attenzione si sia concentrata poco sulle opzioni farmacologiche, privilegiando lo studio delle strategie preventive e, tra le opzioni terapeutiche, il confronto tra tecniche strumentali e chirurgiche.

\section{Costo-efficacia delle strategie di prevenzione primaria}

Saab e coll. hanno sviluppato un modello per l'analisi decisionale basato sui costi e gli esiti di tre strategie alternative per la profilassi dell'emorragia da rottura di varici nel paziente cirrotico: prevenzione primaria con beta-bloccanti in tutti i cirrotici, endoscopia diagnostica con somministrazione dei farmaci solo ai pazienti in cui sono state evidenziate varici di grandi dimensioni, e nessuna profilassi. Rispetto alla rinuncia alla prevenzione, la profilassi dei soli pazienti con evidenza endoscopica di varici di grandi dimensioni è risultata costoefficace, con un costo di 800 \$ per QALY (quality-adjusted life year - anno di vita ponderato per la qualità) guadagnato (scenario base), o addirittura capace di indurre risparmio sui costi sanitari, a seconda delle ipotesi adottate per i parametri più incerti. Sia nello scenario base, sia negli scenari alternativi prospettati con le analisi di sensibilità, l'adozione di una strategia universale di profilassi con beta-bloccanti domina le alternative, ossia produce migliori risultati clinici ad un costo inferiore [8].

Uno studio simile è stato condotto anche da Spiegel e coll., che come outcome clinico hanno però scelto il numero di emorragie da rottura di varice evitate invece dei QALY. L'analisi ha messo in evidenza che la rinuncia alla profilassi è l'opzione meno costosa, ma anche la meno efficace. Rispetto a questa strategia, l'adozione della profilassi universale con betabloccanti in tutti i cirrotici ha un basso (buon) rapporto costo-efficacia: ogni emorragia evitata ha un costo medio di circa 12.000 euro. Quest'ultima strategia è dominante rispetto alle alternative che prevedono lo screening selettivo sui pazienti considerati ad alto rischio a priori, che sono dunque opzioni da scartare; le strategie di screening universale, infine, sono più efficaci della profilassi universale, ma si caratterizzano per un rapporto costo-efficacia sfavorevole, richiedendo un investimento di oltre $175.000 \$$ per evitare un episodio emorragico [38].

Risultati parzialmente simili sono stati ottenuti da un terzo gruppo di ricerca che si è occupato del medesimo problema: il modello markoviano di Arguedas e coll. suggerisce di differenziare la gestione del paziente cirrotico in base allo stato di compenso o meno. Infatti, secondo questi autori, la strategia migliore per i cirrotici compensati si dovrebbe basare sullo screening endoscopico, con un costo decisamente accettabile di $3.605 \$$ per anno di vita salvato, mentre nel cirrotico scompensato la strategia col miglior rapporto costo-efficacia risulta la profilassi universale con beta-bloccanti, con cui ogni anno di vita salvato costa mediamente 1.154 \$ [39].

Alla luce dei loro risultati, gli autori di questi lavori mettono in discussione le raccomandazioni contenute nelle linee guida, che prevedono l'esecuzione di un endoscopia digestiva a tutti i pazienti cirrotici.

Vari studi hanno affrontato il tema della convenienza del monitoraggio emodinamico delle pressioni sanguigne epatiche mediante cateterismo per valutare la risposta alla profilassi farmacologica primaria o per guidare la decisione sulle strategie di prevenzione secondaria, con risultati ampiamente discordanti.

Per quanto riguarda la valutazione della risposta alla profilassi con beta-bloccanti, lo studio di Hicken e coll. indica che il monitoraggio del gradiente pressorio epatico è una strategia molto costosa e difficilmente sostenibile dal servizio o dall'assicurazione sanitaria (108.000$720.000 \$$ per emorragia evitata) [40], mentre i risultati del modello di Imperiale e coll. suggeriscono che il monitoraggio induca risparmi netti o sia per lo meno conveniente $(5.200 \$$ / anno di vita salvato) [41].

L'opportunità di decidere la strategia di profilassi secondaria del sanguinamento da varice in base al monitoraggio emodinamico è stata analizzata da Targownik e coll. in Canada e da Raines et al. negli Usa. I risultati dei due lavori concordano nell'indicare la superiorità clinica del monitoraggio rispetto alla terapia empirica, ma differiscono notevolmente nei rapporti costo-efficacia calcolati, anche in funzione del differente contesto sanitario in cui sono ambientate le simulazioni $[42,43]$. I risultati di queste analisi sono fortemente sensibili alle assunzioni postulate in fase di costruzione del modello teorico, come il tasso di recidiva e l'aspettativa di vita della coorte di pazienti, nonché ai fattori di costo locali. 
Costo-efficacia delle opzioni terapeutiche e di prevenzione secondaria

Nell'ambito di uno studio randomizzato prospettico di confronto tra efficacia e sicurezza della scleroterapia (ES) o della legatura con elastico (EL) nella prevenzione secondaria del sanguinamento da rottura di varici, Gralnek e coll. hanno stimato il consumo di risorse e la costo-efficacia delle due tecniche endoscopiche. ES ed EL non hanno mostrato differenze significative negli outcome clinici principali, il tasso di recidiva e la sopravvivenza a un anno, né nel tasso di obliterazione delle varici, nella durata del ricovero ospedaliero, nel fabbisogno di trasfusioni sanguigne o di ricorrere allo shunt. EL è risultata associata a un'incidenza di fallimento del controllo dell'emorragia attiva significativamente superiore, mentre la formazione di stenosi esofagee è stata significativamente più frequente con ES. I costi sanitari diretti mediani sono risultati simili nei due gruppi di pazienti, per cui anche i rapporti costo-efficacia calcolati per i due outcome principali non sono risultati significativamente differenti. Nel sottogruppo di pazienti con sanguinamento attivo durante la procedura endoscopica, invece, ES è risultato associato a un costo/sopravvissuto nettamente inferiore ( $\$ 28.523 \mathrm{vs.}$ $\$ 51.696)$, in virtù della nota superiorità clinica di questa tecnica in presenza di emorragia acuta. Gli autori ritengono pertanto che la scelta tra le due tecniche endoscopiche alternative debba essere guidata da considerazioni prettamente cliniche, visto il paragonabile assorbimento di risorse sanitarie, per quanto auspichino la conduzione di ulteriori studi di rilevamento degli esiti clinici ed economici delle terapie dell'emorragia da rottura di varice [44].

Questo auspicio, di per sé condivisibile, accomuna gran parte dei ricercatori che si sono occupati dei riflessi economici delle scelte terapeutiche in questa popolazione di pazienti; in effetti, quasi tutti gli studi farmacoeconomici in questo ambito sono stati condotti con l'utilizzo di modelli teorici, anche a causa della notevole varietà delle scelte prospettabili, che richiederebbero a uno studio clinico di confronto tra le modalità princi- pali un disegno molto complesso e un numero considerevole di pazienti arruolati. Esiste comunque qualche eccezione, come quella appena riportata e quella di Meddi et al. ,di cui riassumiamo brevemente i risultati.

Un gruppo di 38 pazienti cirrotici è stato trattato con TIPSS $(n=18)$ o ES $(n=20)$ per prevenire una recidiva di sanguinamento e seguito per 18 mesi, con rilevazione dei costi sanitari assorbiti. Nonostante il tasso di recidiva sia risultato significativamente più alto nei pazienti trattati con ES, il numero totale di giorni di ospedalizzazione è stato simile, a causa del maggior numero di ricoveri per encefalopatia epatica e stenosi dello stent nei pazienti TIPSS. Il costo cumulativo è risultato superiore per il gruppo TIPSS, a causa dei superiori costi dell'intervento; quando il costo è stato calcolato in base all'efficacia (costo/mese libero da emorragia) le differenze non sono risultate significative [45].

Il confronto delle prestazioni cliniche ed economiche di TIPSS e ES è stato svolto anche da Russo e coll. mediante la costruzione di un modello di analisi decisionale ad hoc, alimentato con parametri clinici derivanti dalla letteratura medica internazionale e variabili di costo ottenute da due centri ospedalieri contattati dagli autori. Anche in questo caso, i principali vantaggi della TIPSS derivano dal minor tasso di recidiva, gli svantaggi dalla tendenza a indurre encefalopatia epatica e dai maggiori costi iniziali. L'ipotetica coorte di pazienti era composta da cirrotici in cui il primo episodio di sanguinamento era stato efficacemente controllato con ES e per cui andava scelto il metodo di prevenzione secondaria tra le seguenti alternative: ES profilattica (+ propranololo), TIPSS, o EL. La simulazione del modello ha prodotto i seguenti risultati (tabella II): mortalità paragonabile nei tre gruppi; 0,39 episodi emorragici per paziente per anno con ES, 0,32 con EL e 0,07 con TIPSS; costi totali per paziente per anno di \$23.459, \$23.111 e \$26.275 con ES, EL e TIPSS, rispettivamente. A partire da questi esiti, sono stati calcolati i rapporti costo-efficacia incrementali della tecnica più efficace e più costosa, la TIPSS, rispetto alle alternative: \$8.803 e \$ 12.660 per emorragia evitata rispetto a EL ed ES, rispettivamente (tabella II). In questo modello, inoltre, EL è risul-
Tabella II

Principali risultati dello studio di confronto ES vs. EL vs. TIPSS [46]

\begin{tabular}{lccc} 
& ES & EL & TIPSS \\
\hline Incidenza recidive & 0,39 & 0,32 & 0,07 \\
Costi annuali $(\$)$ & 23.459 & 23.111 & 26.275 \\
ICER TIPSS $(\$ / E E)$ & 8.803 & 12.660 & $/$ \\
\hline
\end{tabular}

ICER TIPSS = Rapporto di costo-efficacia incrementale della TIPSS rispetto alle metodiche endoscopiche, ossia costo aggiuntivo per evento evitato, $\mathrm{EE}=$ evento evitato 


\begin{tabular}{lcccc}
\hline \multicolumn{1}{c}{ Strategia } & $\begin{array}{c}\text { Costi } \\
\text { (migliaia \$) }\end{array}$ & QALY & VH & $\begin{array}{c}\text { Sopravvivenza a } \\
\mathbf{3} \text { anni (\%) }\end{array}$ \\
\hline EBL + MED & 15,1 & 1,68 & 0,13 & 82 \\
MED & 15,7 & 1,57 & 0,77 & 71 \\
EBL & 16,6 & 1,67 & 0,36 & 77 \\
OBS & 21,1 & 1,44 & 1,56 & 56 \\
TIPSS & 30,9 & 1,63 & 0,23 & 77 \\
\hline
\end{tabular}

$\mathrm{EBL}=$ legatura endoscopica con elastico, $\mathrm{MED}=$ terapia medica con beta-bloccanti OBS = osservazione, TIPSS = shunt porto-sistemico intraepatico, QALY = quality adjusted life year - anno di vita ponderato per la qualità, $\mathrm{VH}=$ variceal bleeding - recidiva emorragica

tata dominare ES, essendo associata contemporaneamente a una minor frequenza di emorragia e a costi minori [46].

La superiorità profilattica di EL rispetto $\mathrm{a}$ ES, dimostrata anche dalla meta-analisi di Laine e Cook del 1995 [47], è uno dei presupposti del recente studio di costo/utilità delle strategie di prevenzione secondaria dell'emorragia da varice condotto da Rubenstein e coll. L'obiettivo dello studio era di calcolare e confrontare il costo per QALY delle diverse strategie e di analizzare l'influenza dell'aderenza al trattamento prescritto sul costo/utilità delle singole opzioni. A tal fine, è stato costruito un modello di analisi decisionale a struttura semi-markoviana per confrontare 5 tecniche di profilassi: mera osservazione (OBS), terapia medica (beta-bloccanti-MED), legatura elastica delle varici (EBL), $\mathrm{EBL}+$ terapia medica (EBL + MED) e TIPSS. La simulazione del percorso clinico della coorte di pazienti ha indicato che, secondo le ipotesi formulate per lo scenario di base, la strategia migliore è EBL + MED, che domina tutte le alternative (tabella III).

Su questi risultati sono state condotte approfondite analisi di sensibilità, facendo variare i parametri più incerti in un range di valori plausibili e osservandone l'effetto sui rapporti costo/utilità calcolati. Secondo questi risultati, TIPSS va riservata a quei pazienti in cui è prevedibile un livello molto basso di compliance; negli altri casi i risultati clinici ed economici sono migliori con la legatura endoscopica o la terapia con beta-bloccanti o entrambi [48]. Per chiarire quale di queste tre ultime strategie sia effettivamente la migliore, sono necessari ulteriori studi clinici di confronto che conferiscano maggior robustezza alle ipotesi formulate in fase di modellizzazione del problema.

\section{Collocazione farmacoeconomica di terlipressina}

Come risulta dalla sintetica revisione della letteratura farmacoeconomica pubblicata in materia, non è ancora chiarito quali siano gli approcci più efficaci e convenienti nella gestione del paziente cirrotico a rischio di sanguinamento o risanguinamento da rottura di varici esofagee. Questa incertezza è in parte dovuta alla relativa carenza di dati di efficacia e di consumo di risorse certi, ma è anche secondaria al tipo di paziente interessato, che tipicamente ha prognosi infausta e una aspettativa di vita limitata, in assenza di trapianto d'organo. I trattamenti vasoattivi, tuttavia, appaiono in grado di migliorare la prognosi con un eccesso di spesa relativamente modesto; nel caso di terlipressina, i benefici in termini di mortalità, un outcome particolarmente robusto che difficilmente è distorto da bias, sono risultati evidenti sia quando somministrata da sola, sia quando associata al trattamento endoscopico. Ciò è di particolare importanza, visto che in molti centri clinici questo trattamento viene ormai applicato di routine.

Nei centri clinici in cui l'endoscopia digestiva d'urgenza non è disponibile, la gestione del paziente con sanguinamento attivo richiede la somministrazione tempestiva di un trattamento vasoattivo. Il quesito clinico sulla superiorità di un trattamento farmacologico rispetto agli altri non è ancora stato risolto. La meta-analisi di Ioannou et al., la più completa finora condotta, non ha evidenziato alcuna differenza di efficacia tra somatostatina e terlipressina, mentre indica un lieve vantaggio di octreotide sul tasso di fallimento dell'emostasi. Tuttavia, questa differenza emerge da piccoli studi "a bassa qualità", in cui la presenza di bias è probabile. Inoltre, e a differenza di terlipressina, octreotide non ha mostrato un vantaggio in termini di sopravvivenza rispetto al placebo né in studi singoli né nelle meta-analisi, nonostante siano stati studiati oltre 500 pazienti [49]. Per chiarire in maniera più definitiva quali siano le reali differenze cliniche tra octreotide e terlipressina devono dunque essere ancora progettati e condotti ampi studi randomizzati.

Per la valutazione delle prestazioni farmacoeconomiche di terlipressina nel contesto sanitario italiano sono indubbiamente necessari studi ben disegnati che registrino e
Tabella III

Principali risultati dello studio di costo/utilità di Rubenstein et al. [48] 


\begin{tabular}{|c|c|c|}
\hline Prospettiva & Valore clinico & Valore economico \\
\hline \multicolumn{3}{|l|}{ Paziente } \\
\hline Positivo & $A, B$ & C \\
\hline Negativo & $D^{*}$ & 1 \\
\hline \multicolumn{3}{|l|}{ Medico } \\
\hline Positivo & $A, B, E, F$ & l \\
\hline Negativo & $D^{*}, G$ & l \\
\hline \multicolumn{3}{|l|}{ Ospedale } \\
\hline Positivo & $A, B, E, F$ & $\mathrm{~F}, \mathrm{H}, \mathrm{I}$ \\
\hline Negativo & $D^{*}, G$ & $L^{*}$ \\
\hline \multicolumn{3}{|l|}{ SSN } \\
\hline Positivo & $A, B, E, F$ & $\mathrm{~F}, \mathrm{H}, \mathrm{I}$ \\
\hline Negativo & $D^{*}, G$ & 1 \\
\hline \multicolumn{3}{|l|}{ Società } \\
\hline Positivo & $A, E$ & $C, M^{*}$ \\
\hline Negativo & $D^{*}$ & $L^{*}$ \\
\hline
\end{tabular}

\section{Tabella IV}

Matrice dei valori della terlipressina nelle varie prospettive

$A=$ Riduzione mortalità da emorragia, anche nei pazienti trattati con scleroterapia

$\mathrm{B}=$ Semplicità della somministrazione; non necessita di infusione continua (a differenza degli altri f. vasoattivi)

$\mathrm{C}=$ Riduzione della perdita di produttività grazie alla maggiore sopravvivenza

$\mathrm{D}=$ Casi di reazioni avverse, casi di inefficacia

$\mathrm{E}=$ Controllo dell'emorragia in una buona percentuale di casi

$\mathrm{F}=$ Non richiede competenze specialistiche (a diff. delle tecniche endoscopiche)

$G=$ Possibile maggior rischio di fallimento dell'emostasi rispetto a octreotide

$\mathrm{H}=$ Trattamento efficace ed efficiente

I = Aumento dell'efficacia e dell'efficienza della scleroterapia

$\mathrm{L}=$ Costo del farmaco

$M=$ Posti di lavoro nell'industria farmaceutica

* Si tratta di fattori generali, applicabili a qualsiasi farmaco

analizzino gli esiti clinici ed economici della sua somministrazione, o perlomeno un modello matematico che rispecchi con fedeltà la complessità del problema.

Una tecnica di valutazione farmacoeconomica preliminare, utile a individuare le diverse ricadute potenziali di un trattamento, è la cosiddetta "analisi dei valori”. Poiché le ricadute possono essere diverse per i vari attori coinvolti nelle decisioni sanitarie, sia a livello di utilità che a livello di costi, tale tecnica prevede la costruzione della matrice dei valori, una serie di tabelle $2 \times 2$ che esplicita conseguenze positive e negative di un'alternativa nei due livelli di valutazione, clinico ed economico, per ogni punto di vista rilevante.

La tabella IV presenta un'analisi dei valori dell'utilizzo di terlipressina nei pazienti cirrotici con sanguinamento da rottura di varice, compilata in base alle informazioni cliniche descritte precedentemente.

\section{CONCLUSIONI}

La formazione e la rottura di varici esofagee nei pazienti cirrotici con ipertensione portale comportano un onere clinico ed economico molto rilevante per le società $\mathrm{e} i$ sistemi sanitari. Nonostante siano stati pubblicati oltre 50 trial randomizzati [50] di valutazione dei farmaci vasoattivi nel controllo di queste emorragie, permane tuttora notevole controversia sul loro ruolo e la loro efficacia.
A livello economico l'incertezza è ancora maggiore, vista la differente strutturazione dei sistemi sanitari e la difficoltà a individuare endpoint robusti che permettano di effettuare confronti affidabili.

Terlipressina, un analogo sintetico della vasopressina, ha dimostrato di essere in grado di migliorare gli esiti clinici dei pazienti con emorragia, siano essi trattati con tecniche endoscopiche o no.

In questo lavoro, abbiamo cercato di mettere in evidenza quali siano le caratteristiche farmacologiche cliniche in grado di avere un impatto economico sulla gestione di questa tipologia di pazienti.

Il nostro obiettivo non era quello di svolgere una valutazione analitica delle prestazioni farmacoeconomiche di terlipressina nel contesto sanitario nazionale, sicuramente auspicabile, bensì di delinearne i contorni, anche per mettere in evidenza quali siano i punti interrogativi, clinici ma anche economici, che vanno chiariti per consentire di effettuare scelte razionali in questa patologia dall'alto costo sociale.

Il profilo farmacologico e clinico di terlipressina, anche in confronto con i competitors, è caratterizzato da un ottimo rapporto rischi/benefici e ciò depone per un buon rapporto costo/utilità. Conclusioni definitive in merito, tuttavia, potranno essere tratte solamente con la conduzione di studi metodologicamente corretti che analizzino in dettaglio i costi e le conseguenze delle varie alternative disponibili. 


\section{BIBLIOGRAFIA}

1. D'Amico G, Pagliaro L, Bosch J The treatment of portal hypertension: a meta-analytic review. Hepatology. 1995 Jul;22(1):332-54.

2. Pagliaro L, D'Amico G, Sorensen TI, Lebrec D, Burroughs AK, Morabito A, Tine F, Politi F, Traina M. Prevention of first bleeding in cirrhosis. A meta-analysis of randomized trials of nonsurgical treatment. Ann Intern Med. 1992 Jul 1;117(1):59-70.

3. de Franchis R, Vitagliano P, Agape D, Antoniozzi F, Arcidiacono P, Cipolla M, Falsitta M, Meucci G, Rizzi P, Torgano G, et al. Eradication of esophageal varices by endoscopic sclerotherapy: how much is enough? Gastrointest Endosc. 1988 SepOct;34(5):395-9

4. Schlichting P, Christensen E, Fauerholdt L et al. Main causes of death in cirrhosis. Scand J Gastroenterol 1982; 18:881-8

5. Graham DY, Smith JL. The course of patients after variceal hemorrhage. Gastroenterology. 1981 Apr;80(4):800-9.

6. Zoli M, Cordiani MR, Marchesini G, Iervese T, Labate AM, Bonazzi C, Bianchi G, Pisi E. Prognostic indicators in compensated cirrhosis. Am J Gastroenterol. 1991 Oct;86(10):1508-13.

7. Burroughs AK Surgery and sclerotherapy for treatment of portal hypertension and oesophageal varices. Drugs. 1989;37 Suppl 2:25-9; discussion 47.

8. Saab S, DeRosa V, Nieto J, Durazo F, Han S, Roth B.Costs and clinical outcomes of primary prophylaxis of variceal bleeding in patients with hepatic cirrhosis: a decision analytic model. Am J Gastroenterol. 2003 Apr;98(4):763-70.

9. Merli M, Nicolini G, Angeloni S, Rinaldi V, De Santis A, Merkel C, Attili AF, Riggio O. Incidence and natural history of small esophageal varices in cirrhotic patients. J Hepatol. 2003 Mar;38(3):266-72

10. Wolf DC The management of variceal bleeding: past, present and future. Mt Sinai J Med. 1999 Jan;66(1):1-13.

11. Groszmann RJ. Hyperdynamic circulation of liver disease 40 years later: pathophysiology and clinical consequences. Hepatology. 1994 Nov;20(5):1359-63.

12. Hermann R, Maier KP.Die portal-hypertensive Gastropathie. Schweiz Med Wochenschr 1996; 126:1066-73

13. Forsling ML, Aziz LA, Miller M, Davies R, Donovan B. Conversion of triglycylvasopressin to lysine-vasopressin in man.J Endocrinol. 1980 May;85(2):237-44

14. Nilsson G, Lindblom P, Ohlin M, Berling R, Vernersson E Pharmacokinetics of terlipressin after single i.v. doses to healthy volunteers. Drugs Exp Clin Res. 1990;16(6):307-14

15. Lin HC, Tsai YT, Lee FY, Chang TT, Wang SS, Lay CS, Lee SD, Lo KJ. Systemic and portal haemodynamic changes following triglycyllysine vasopressin plus nitroglycerin administration in patients with hepatitis B-related cirrhosis. J Hepatol. 1990 May;10(3):370-4.

16. Cestari R, Braga M, Missale G, Ravelli P, Burroughs AK. Haemodynamic effect of triglycyl-lysine-vasopressin (glypressin) on intravascular oesophageal variceal pressure in patients with cirrhosis. A randomized placebo controlled trial. J Hepatol. 1990 Mar;10(2):205-10

17. Merkel C, GattaA, Bolognesi M, Finucci G, Battaglia G,Angeli P,Zuin R. Hemodynamic changes of systemic, hepatic, and splenic circulation following triglycyl-lysin-vasopressin administration in alcoholic cirrhosis.Dig Dis Sci. 1988 Sep;33(9):1103-9.

18. Nevens F, Van Steenbergen W, Yap SH, Fevery J. Assessment of variceal pressure by continuous non-invasive endoscopic registration: a placebo controlled evaluation of the effect of terlipressin and octreotide. Gut. 1996 Jan;38(1):129-34.

19. Breuer HW, Charchut S, Worth H, Trampisch HJ, Glanzer K. Endobronchial versus intravenous application of the vasopressin derivative glypressin during diagnostic bronchoscopy.Eur Respir J. 1989 Mar;2(3):225-8.

20. Ioannou G, Doust J, Rockey DC. Terlipressin for acute esophageal variceal hemorrhage.Cochrane Database Syst Rev. 2003;(1):CD002147

21. Freeman JG, Cobden I, Lishman AH, Record CO. Controlled trial of terlipressin ('Glypressin') versus vasopressin in the early treatment of oesophageal varices. Lancet. 1982 Jul 10;2(8289):66-8.

22. Rodriguez-Perez F, Groszmann RJ Pharmacologic treatment of portal hypertension. Gastroenterol Clin North Am. 1992 Mar;21(1):15-40

23. Burroughs AK, McCormick PA, Hughes MD, Sprengers D, D’Heygere F, McIntyre N. Randomized, double-blind, placebocontrolled trial of somatostatin for variceal bleeding. Emergency control and prevention of early variceal rebleeding. Gastroenterology. 1990 Nov;99(5):1388-95.

24. Shields R, Jenkins SA, Baxter JN, Kingsnorth AN, Ellenbogen S, Makin CA, Gilmore I, Morris AI, Ashby D, West CR. $A$ prospective randomised controlled trial comparing the efficacy of somatostatin with injection sclerotherapy in the control of bleeding oesophageal varices.J Hepatol. 1992 Sep;16(1-2):128-37.

25. Sung JJ, Chung SC, Lai CW, Chan FK, Leung JW, Yung MY, Kassianides C, LiAK. Octreotide infusion or emergency sclerotherapy for variceal haemorrhage. Lancet. 1993 Sep 11;342(8872):637-41.

26. Planas R, Quer JC, Boix J, Canet J, Armengol M, Cabre E, Pintanel T, Humbert P, Oller B, Broggi MA, et al. A prospective randomized trial comparing somatostatin and sclerotherapy in the treatment of acute variceal bleeding. Hepatology. 1994 Aug;20(2):370-5. 
27. McCormick PA, Chin J, Greenslade L, Karatapanis S, Dick R, McIntyre N, Burroughs AK. Cardiovascular effects of octreotide in patients with hepatic cirrhosis. Hepatology. 1995 May;21(5):1255-60.

28. Escorsell A, Ruiz del Arbol L, Planas R, Albillos A, Banares R, Cales P, Pateron D, Bernard B, Vinel JP, Bosch J Multicenter randomized controlled trial of terlipressin versus sclerotherapy in the treatment of acute variceal bleeding: the TEST study. Hepatology. 2000 Sep;32(3):471-6.

29. Ganne-Carrie N, Hadengue A, Mathurin P, Durand F, Erlinger S, Benhamou JP. Hepatorenal syndrome. Long-term treatment with terlipressin as a bridge to liver transplantation. Dig Dis Sci. 1996 Jun;41(6):1054-6.

30. Cervoni JP, Lecomte T, Cellier C, Auroux J, Simon C, Landi B, Gadano A, Barbier JP. Terlipressin may influence the outcome of hepatorenal syndrome complicating alcoholic hepatitis. Am J Gastroenterol. 1997 Nov;92(11):2113-4.

31. Hadengue A, Gadano A, Moreau R, Giostra E, Durand F, Valla D, Erlinger S, Lebrec D. Beneficial effects of the 2-day administration of terlipressin in patients with cirrhosis and hepatorenal syndrome. J Hepatol. 1998 Oct;29(4):565-70.

32. Uriz J, Gines P, Cardenas A, Sort P, Jimenez W, Salmeron JM, Bataller R, Mas A,Navasa M, Arroyo V, Rodes J.Terlipressin plus albumin infusion: an effective and safe therapy of hepatorenal syndrome. J Hepatol. 2000 Jul;33(1):43-8.

33. Ortega R, Gines P, Uriz J, Cardenas A, Calahorra B, De Las Heras D, Guevara M,Bataller R, Jimenez W, Arroyo V, Rodes J.Terlipressin therapy with and without albumin for patients with hepatorenal syndrome: results of a prospective, nonrandomized study. Hepatology. 2002 Oct;36(4 Pt 1):941-8.

34. Alessandria C, Venon WD, Marzano A, Barletti C, Fadda M, Rizzetto M. Renal failure in cirrhotic patients: role of terlipressin in clinical approach to hepatorenal syndrome type 2. Eur J Gastroenterol Hepatol. 2002 Dec;14(12):1363-8.

35. Saner F, Kavuk I, Lang H, Biglarnia R, Fruhauf NR, Schafers RF, Malago M,Broelsch CE. Terlipressin and gelafundin: safe therapy of hepatorenal syndrome. Eur J Med Res. 2004 Feb 27;9(2):78-82.

36. Panes J, Pique JM, Bordas JM, Llach J, Bosch J, Teres J, Rodes J. Reduction of gastric hyperemia by glypressin and vasopressin administration in cirrhotic patients with portal hypertensive gastropathy.Hepatology. 1994 Jan;19(1):55-60.

37. Vachiery F, Moreau R, Gadano A, Yang S, Sogni P, Hadengue A, Cailmail S, Soupison T, Lebrec D. Hemodynamic and metabolic effects of terlipressin in patients with cirrhosis receiving a nonselective beta-blocker.Dig Dis Sci. 1996 Sep;41(9):1722-6

38. Spiegel BM, Targownik L, Dulai GS, Karsan HA, Gralnek IM. Endoscopic screening for esophageal varices in cirrhosis: Is it ever cost effective? Hepatology. $2003 \mathrm{Feb} ; 37(2): 366-77$.

39. Arguedas MR, Heudebert GR, Eloubeidi MA, Abrams GA, Fallon MB.Cost-effectiveness of screening, surveillance, and primary prophylaxis strategies for esophageal varices. Am J Gastroenterol. 2002 Sep;97(9):2441-52.

40. Hicken BL, Sharara AI, Abrams GA, Eloubeidi M, Fallon MB, Arguedas MR. Hepatic venous pressure gradient measurements to assess response to primary prophylaxis in patients with cirrhosis: a decision analytical study. Aliment Pharmacol Ther. 2003 Jan; 17(1):145-53.

41. Imperiale TF, Chalasani N, Klein RW. Measuring the hemodynamic response to primary pharmacoprophylaxis of variceal bleeding: a cost-effectiveness analysis. Am J Gastroenterol. 2003 Dec;98(12):2742-50.

42. Targownik LE, Spiegel BM, Dulai GS, Karsan HA, Gralnek IMThe cost-effectiveness of hepatic venous pressure gradient monitoring in the prevention of recurrent variceal hemorrhage. Am J Gastroenterol. 2004 Jul;99(7):1306-15.

43. Raines DL, Dupont AW, Arguedas MR.Cost-effectiveness of hepatic venous pressure gradient measurements for prophylaxis of variceal re-bleeding. Aliment Pharmacol Ther. 2004 Mar 1;19(5):571-81.

44. Gralnek IM, Jensen DM, Kovacs TO, Jutabha R, Machicado GA, Gornbein J, King J, Cheng S, Jensen ME.The economic impact of esophageal variceal hemorrhage: cost-effectiveness implications of endoscopic therapy. Hepatology. 1999 Jan;29(1):44-50.

45. Meddi P, Merli M, Lionetti R, De Santis A, Valeriano V, Masini A, Rossi P, Salvatori F, Salerno F, de Franchis R, Capocaccia L, Riggio O. Cost analysis for the prevention of variceal rebleeding: a comparison between transjugular intrahepatic portosystemic shunt and endoscopic sclerotherapy in a selected group of Italian cirrhotic patients. Hepatology. 1999 Apr;29(4):1074-7.

46. Russo MW, Zacks SL, Sandler RS, Brown RS.Cost-effectiveness analysis of transjugular intrahepatic portosystemic shunt (TIPS) versus endoscopic therapy for the prevention of recurrent esophageal variceal bleeding. Hepatology. 2000 Feb;31(2):358-63.

47. Laine L, Cook D. Endoscopic ligation compared with sclerotherapy for treatment of esophageal variceal bleeding. A metaanalysis. Ann Intern Med. 1995 Aug 15;123(4):280-7.

48. Rubenstein JH, Eisen GM, Inadomi JM. A cost-utility analysis of secondary prophylaxis for variceal hemorrhage.Am J Gastroenterol. 2004 Jul; $99(7): 1274-88$.

49. Corley DA, Cello JP, Adkisson W, Ko WF, Kerlikowske K Octreotide for acute esophageal variceal bleeding: a metaanalysis.Gastroenterology. 2001 Mar;120(4):946-54.

50. Sharara AI, Rockey DC.Gastroesophageal variceal hemorrhage. N Engl J Med. 2001 Aug 30;345(9):669-81. 